\title{
Selected aspects of endothelial dysfunction and their influence on the atherosclerosis process modeled and analyzed by Petri net based approach
}

\author{
DOROTA FormanowiCZ $^{1 *}$, AdAM KOZAK ${ }^{2}$, PIOTR FormanowICZ ${ }^{2,3}$ \\ ${ }^{1}$ Department of Clinical Biochemistry and Laboratory Medicine, Poznan University of Medical Sciences, Poznan, Poland \\ ${ }^{2}$ Institute of Computing Science, Poznan University of Technology, Poznan, Poland \\ ${ }^{3}$ Institute of Bioorganic Chemistry, Polish Academy of Sciences, Poznan, Poland \\ * Corresponding author: doforman@ump.edu.pl
}

\begin{abstract}
In this paper a Petri net based model of the process of endothelial dysfunction is presented. The model describes some selected aspects of this process and their relation with atherosclerosis. Models expressed in the language of Petri net theory have, on the one hand, an intuitive graphical interpretation, and on the other hand, they can be analyzed using rigorous mathematical methods. Such analysis and simulation of the net behavior can be supported by many freely available software tools. Here, results of an analysis based on t-invariants of the proposed model are presented. In particular, feasible t-invariants, MCT-sets and t-clusters have been calculated and their biological meaning has been determined.
\end{abstract}

Key words: endothelial dysfunction, biological systems, modeling, Petri nets, t-invariants

\section{Introduction}

Understanding of the atherosclerosis process has evolved beyond the view that atherosclerotic lesions consist of a lifeless collection of lipid debris. Current evidence supports a central role for inflammation and immunological response in all phases of the atherosclerotic plaque formation. Endothelial dysfunction plays a key role in these phenomena and is characterized by reduced vasodilation, a proinflammatory state, and prothrombic properties (Endemann and Schiffrin, 2004). These disorders ultimately allow lipids and toxins to penetrate the endothelial layer and enter the smooth muscle cells resulting in the initiation of an oxidative stress and inflammatory cascade that culminates in the development of atherosclerotic plaque deposits. Subsequently, these plaques begin to calcify and, over the time, become prone to rupture. If a plaque deposit ruptures, the result is oftentimes a fatal blood clot.

Numerous factors, such as hypercholesterolemia, hypertension, and insulin resistance, which directly contribute to endothelial dysfunction whose hallmark is an impaired endothelium-dependent vasodilation mediated by nitric oxide (NO), have been identified. Thus, the reduced bioavailability of $\mathrm{NO}$ has been proposed as a major mechanism of endothelial dysfunction and a contributor to the initiation, progression and complications of atherosclerosis.

The process of endothelial dysfunction is very complex and not fully understood yet. Although new discoveries continuously improve the theories concerning this process.

It is becoming more and more clear that complexity is one of the most fundamental properties of living organisms. This means, among others, that their structure and functionality results not only directly from the properties of their basic building blocks but also from the dense network of interactions among these elementary units. From this it follows that studying these interactions is necessary for a deep understanding of biological processes. This, in turn, leads to the systems approach to studying biological phenomena, according to which they are seen and analyzed as complex systems. Such an approach has been, for years, developed and applied in the area of technical sciences and as a result 
advanced systems theories have been formulated. They concern systems as mathematical objects but the theoretical results may be applied for studying real physical, technical, biological and other systems. Obviously, each of these areas has some specificity, so usually the general theories should be adapted to a particular area of applications. Biological systems are probably the most complex ones studied so far, hence, their analysis is often more challenging than in the case of technical systems.

The first and necessary step in systems approach is building a precise model of the analyzed system. The model is formulated using the language of some branch of mathematics. One of them is the theory of Petri nets. Such nets were proposed in the early 1960 s by Carl A. Petri in the context of computer systems (Petri, 1962). For years they have been applied to model and analyze concurrent stochastic technical systems, especially the telecommunication and computer systems. But with the rapid development of computational biology in the last two decades, Petri nets are also being used for modeling and analysis of biological processes.

Petri net based models have, on the one hand, an intuitive graphical representation, and on the other hand, they can be analyzed using formal mathematical methods. Moreover, many software tools supporting such an analysis and allowing simulation of the nets are freely available. The models expressed in the language of Petri net theory are the qualitative ones, what allows to catch the structure of the biological system and at the same time allows to avoid the necessity of determining the exact values of parameters needed for models based on differential equations. However, there exist many extensions of Petri nets that can be used for extending the qualitative models by adding quantitative information of various types. This makes Petri nets a very effective and promising method for modeling and analyzing biological systems (cf. Koch et al., 2011).

In this paper, a Petri net based model of selected aspects of the process of endothelial dysfunction is formulated and analyzed. In addition, relations of this process with atherosclerosis are also taken into account in the model.

\section{Petri nets}

In this section, elements of Petri net theory are briefly presented. First, the informal definition of the nets is provided, then they are formally defined and some methods of the analysis of their properties are described. These methods will then be used in the next section in the analysis of the proposed model.

The structure of a Petri net is based on a directed bipartite graph. Such graphs are composed of vertices of two kinds, which in the context of Petri nets are called places and transitions. Each arc of the graph connects two vertices of different types. The places correspond to some passive system components (e.g. molecules) while transitions model active system elements (e.g. chemical reactions). The arcs describe some casual relations between passive and active system components. Places, transitions and arcs compose the structure of a Petri net, however, they do not provide any dynamics being the fundamental property of these nets. This dynamics is brought into the net by tokens residing in places. They correspond to resources of a system, e.g. in the case of biochemical or biological systems, tokens residing in a place corresponding to some compound may indicate the quantity of this compound present in a particular part of the system. The tokens flow from places through transitions to another places. The rule controlling this flow is simple and fundamental for the behavior of Petri nets. Each transition $t_{j}$ has some input places $p_{i}$ (these places are starting vertices of arcs whose ending vertex is the considered transition, i.e. the ones for which there exists $\left.\operatorname{arc}\left(p_{i}, t_{j}\right)\right)$ and some output places $p_{k}$ (such places are ending vertices of some arcs whose starting vertex is transition $t_{j}$, i.e. the places for which $\operatorname{arc}\left(t_{j}, p_{k}\right)$ exists in the net). The arcs are labeled by positive integer numbers called weights. When in every input place of a given transition the number of tokens residing in the place is greater than or equal to the weight of an arc connecting this place with the transition, the transition becomes enabled. An enabled transition can be fired what means that the tokens can flow from its input places to its output places. The number of tokens flowing via a given arc is equal to the weight of that arc. The distribution of tokens over all places at a particular time corresponds to the system state at this time and is called marking. Usually of particular importance is the initial marking, i.e. the one before any transition was fired. It corresponds to the initial system state. In a graphical representation of a Petri net the places are depicted as circles, the transitions as rectangles, the arcs as arrows and the tokens as dots or numbers. The arcs 
whose weights are equal to one usually are not explicitly labeled by this weight in the graphical representation of the net (cf. Reisig, 1985; Murata, 1989; Sackmann et al., 2007; Reisig, 2011).

A Petri net can be formally defined as follows ( $\mathrm{Mu}$ rata, 1989):

\section{DEFINITION}

A Petri net is a 5 -tuple $Q=\left(P, T, F, W, M_{0}\right)$, where: $P=\left\{p_{1}, p_{2}, \ldots, p_{n}\right\}$ is a finite set of places,

$T=\left\{t_{1}, t_{2}, \ldots, t_{m}\right\}$ is a finite set of transitions,

$F \subseteq(P \times T) \cup(T \times P)$ is a set of arcs,

$W: F \rightarrow Z^{+}$is a weight function,

$M_{0}: P \rightarrow \mathrm{N}$ is an initial marking,

$P \cap T=\varnothing \wedge P \cup T \neq \varnothing$.

There are two special kinds of transitions, i.e. input transitions and output transitions. There are no input places for input transitions and no output places for output transitions. From this follows that input transitions are active all the time and output transitions, when fired, do not produce any tokens. Transitions of these types can model the interactions of the system with its environment.

The graphical representation of a Petri net is very intuitive and useful for simulation of its behavior but it cannot be a basis for the analysis of the formal properties of the net. For this purpose, another representation, called incidence matrix, is used. In such a matrix $A=\left(a_{i j}\right)_{n \times m}$ rows correspond to places while columns correspond to transitions. The matrix entry $a_{i j}$ is an integer which is equal to the difference between the number of tokens residing in place $p_{i}$ before and after firing transition $t_{j}$ (Murata, 1989; Reisig, 2011).

Obviously (almost) every model is constructed in order to analyze the properties of the modeled object on the basis of the results of the model analysis. In the case of Petri net based models of biological systems, the analysis can be mainly based on invariants. The invariants correspond to some parts of the net that have some welldefined properties. When the model is properly constructed they should also correspond to some functional subunits of the modeled system. There are two kinds of invariants, i.e. p-invariants (place invariants) and t-invariants (transitions invariants).

A p-invariant is vector $y \in \mathbb{N}^{\mathrm{n}}$ satisfying the equation (Murata, 1989; Sackmann et al., 2007; Sackmann, 2011):

$$
A^{T} \cdot y=0
$$

Analogously, a t-invariant is vector $x \in \mathbb{N}^{m}$ satisfying the equation (Murata, 1989; Sackmann et al., 2007; Sackmann, 2011):

$$
A \cdot x=0
$$

A p-invariant corresponds to a set of places over which a weighed sum of tokens is constant, while a t-invariant corresponds to a set of transitions whose firing, a required number of times, does not change the marking of the net.

A Petri net should be covered by invariants, especially by t-invariants. This means that every transition (in the case of t-invariants) should belong to some invariant. The transitions which are not contained with any t-invariant do not influence the net behavior. When the net is a model of some (biological) system it indicates that there is an error in this model.

An invariant is minimal if it does not contain any other invariant. Since any invariant can be obtained as a linear combination of some minimal invariants, it is sufficient to consider only the minimal ones. From the biological point of view, feasible t-invariants are especially interesting (Sackmann et al., 2006; Sackmann et al., 2007; Sackmann, 2011). An invariant of this type is a minimal t-invariant so that all its transitions can be fired without firing any transition which does not belong to this invariant. The reason why a minimal t-invariant is not feasible is an occurrence of read arcs in the net. These arcs are not reflected in the incidence matrix. If a transition is connected by a read arc with its input place it means that the firing of this transition does not remove the tokens from this place (this is the reason that these arcs are not reflected in the incidence matrix). Such arcs can be used to model an activation of a chemical reaction by the catalyst (which is necessary for the reaction to occur but it is not consumed in the reaction).

If Petri net is a model of a biological system then the properties of the net should be correlated with the properties of the modeled system. In particular, every t-invariant should have some biological meaning, i.e. it should correspond to some functional subunit of the biological system. However, determining such a biological interpretation of every minimal t-invariant may be difficult in the case when the number of these invariants is large. In such a case, an analysis of some other groups of transitions may be helpful. In particular, they can be grouped into so-called Maximal Common Transition sets 
(MCT-sets) which contain transitions being elements of exactly the same feasible t-invariants. These sets are disjoint and correspond to a partition of the modeled biological system into some functional units whose biological meaning should be determined (Sackmann et al., 2006; Sackmann et al., 2007; Sackmann, 2011; Formanowicz et al., 2011).

Another analytical method is based on grouping the feasible $t$-invariants into so-called t-clusters. Such a clustering is based on similarity between invariants. Here, in general, various similarity measures can be used. For the construction of t-clusters, standard clustering algorithms such as UPGMA can be applied (Sackmann et al., 2007; Grafahren-Belau et al., 2008; Sackmann, 2011; Formanowicz et al., 2011).

MCT-sets and t-clusters can also be calculated in the cases where the number of feasible t-invariants is moderate. Even in these cases, analysis of these objects may be helpful in understanding the behavior of the modeled biological system.

\section{Results and discussion}

Firstly, in this section, the process of endothelial dysfunction is described in an informal way. Next, the formal model based on Petri net is presented and analyzed.

Informal description of the selected aspects of endothelial dysfunction and their influence on atherosclerotic plaque formation

The vascular endothelium, by secretion mediators that influence vascular hemodynamics, plays a pivotal role in regulating blood flow and is responsible for maintaining the balance between vasodilatation and vasoconstriction, between inhibition and stimulation of the smooth muscle cells proliferation and migration, and between fibrinolysis and thrombogenesis (de Haro Miralles et al., 2009).

Many factors may lead to the endothelial imbalance, among the most important of which is an inflammatory process mediated, among others, by proinflammatory cytokines. When this balance is disturbed it comes to an endothelial dysfunction, which is thought to be a key event in the development of the atherosclerotic process. As a result of endothelial dysfunction, which occurs in different disease states, the diminished synthesis of nitric oxide (NO) appears. Because of NO function, an abnormal production of it can adversely affect blood flow and other vascular functions.

Under normal conditions, NO is synthesized by the enzymatic action of nitric oxide synthase (NOS) from its precursor L-arginine and stimulates soluble guanylate cyclase (sGC), thereby increasing cyclic guanosine monophosphate (cGMP) levels thus leading to a transient drop in cellular calcium and vasorelaxation.

Two endothelial forms of NOS are distinguished, i.e. constitutive NOS (eNOS, type III) and inducible NOS (iNOS, type II). Under normal and basal conditions in blood vessels, NO is continually produced by eNOS, whose activity is calcium and calmodulin $(\mathrm{CaM})$ dependent. The constitutive eNOS is a multi-domain enzyme consisting of an N-terminal oxygenase that contains binding sites for heme, L-arginine (Cheng et al., 1997) and tetrahydrobiopterin $\left(\mathrm{BPH}_{4}\right)$ and a reductase domain containing binding sites for flavin adenine dinucleotide (FMN), flavin mononucleotide (FAD), reduced nicotinamide adenine dinucleotide phosphate (NADPH) and CaM (Sessa et al., 1992).

There are two basic pathways for the eNOS stimulation, both of which involve a release of calcium ions from storage sites. First, the shearing forces (increased shear stress) acting on the vascular endothelium generated by blood flow cause a release of calcium and subsequent eNOS activation. Therefore, increase in blood flow stimulates NO formation (flow-dependent NO formation). Second, endothelial receptors for a variety of ligands, for example acetylocholine (Ach), vasodilator - bradykinin, $\mathrm{BPH} 4, \mathrm{NADPH}$, can stimulate calcium release and subsequent NO production (receptor-stimulated NO formation). The activity of eNOS is regulated by its subcellular localization, phosphorylation and through its interaction with different proteins. The interaction of eNOS with caveolin appears to serve as a key determinant of the enzyme activity and this interaction may be dynamically regulated by the $\mathrm{Ca}^{2+}$-CaM-dependent activation of NOS in endothelial cells. The association of eNOS with caveolin-1 is believed to maintain eNOS at an inactive state and the increased intercellular calcium level in response to vasodilator agonists or shear stress displaces the inhibitor caveolin-1 from CaM, activating eNOS (Davignon and Ganz, 2004).

The second endothelial form of NOS - iNOS has been described as calcium-insensitive, likely due to its tight non-covalent interaction with $\mathrm{CaM}$ and $\mathrm{Ca}^{2+}$. Under nor- 
mal, basal conditions, the activity of iNOS is very low. However, iNOS activity is stimulated during inflammation by bacterial endotoxins (e.g., lipopolysaccharide) and cytokines such as tumor necrosis factor (TNF) and interleukins. Thus, the induction of the high-output iNOS usually occurs in an oxidative environment and high levels of NO have the opportunity to react with superoxide, leading to peroxynitrite $\left(\mathrm{OONO}^{-}\right)$formation and cell toxicity. When NO is formed by vascular endothelium it rapidly diffuses into the blood where it binds to hemoglobin and is subsequently broken down. It also diffuses into the vascular smooth muscle cells adjacent to the endothelium where it binds to and activates guanylyl cyclase, which induces smooth muscle relaxation. This is done in three different ways. One of them is an increased intracellular cGMP which inhibits calcium entry into the cell and decreases intracellular calcium concentrations. The second is an activation of $\mathrm{K}^{+}$channels, which leads to hyperpolarization and relaxation, and the third is the cGMP-dependent protein kinase stimulation that activates myosin light chain phosphatase and leads to smooth muscle relaxation.

The processes resulting in NO decrease contribute to the lowering of an active uptake of calcium, which results in vasoconstriction of the vessels and finally increases the atherosclerosis process.

Among the processes that reduce NO are those which are stimulated by an asymmetric dimethylarginine (ADMA) - a naturally occurring component of human blood plasma. It is formed as a metabolic byproduct of continuous protein turnover in all cells of the body. Under the action of an enzyme called methionine-dependent protein arginine N-methyltransferase (PRMT), ADMA is formed by methylation of arginine residues of nuclear proteins. More than one decade ago, ADMA was first reported to exert biological effects by inhibiting NO synthesis (Vallance et al., 1992) by competing with the substrate of this enzyme, L-arginine. Upon hydrolysis, ADMA residues are released to pass into blood and are available for binding NOS to modulate the rate of NO production. A principal mechanism of regulating ADMA concentrations is through the action of the catabolic enzyme dimethylarginine dimethylaminohydrolase (DDAH) that degrades ADMA to dimethylamine and citrulline, which is essential for the synthesis of arginine - a key substrate for NO synthesis. The elevated levels of ADMA are associated with endothelial dysfunction and atherosclerosis (Cooke, 2000).

Oxidative stress has been shown to contribute in endothelial dysfunction and atherosclerosis development. It may decrease the bioavailability and the bioactivity of NO. Under its influence, peroxynitrite $\left(\mathrm{ONOO}^{-}\right)$is formed. Besides, the inflammatory process and the oxidative stress stimulate endothelium for the synthesis of many factors that play an important role in atherosclerotic process. Among others, they include vascular cell adhesion protein-1 (VCAM-1), inter-cellular adhesion molecule-1 (ICAM-1), selectins $\mathrm{P}$ and $\mathrm{E}$, platelet-activating factor $(\mathrm{PAF})$, reactive oxygen species (ROS) and vasoconstrictors: endothelin and tromboxane. ICAM-1 together with VCAM-1, CD31 receptor and intracellular proteins participate in the cascade and in the coordination of adhesion events between leukocytes and the activated endothelial cells (Zhang et al., 2010). When endothelial cells are activated during inflammation, P-selectin moves from an internal cell location to the endothelial cell surface and along with adhesion molecules P-selectin glycoprotein ligand-1 (PSGL-1) plays an essential role in the primary interactions between leukocytes, both endothelial cells and activated platelets and in the initial recruitment of leukocytes. When endothelial cells or platelets are activated, P-selectin is rapidly translocated to the cell surface through the fusion of vesicles and the plasma membranes and is exposed on the external plasma membranes of endothelial cells and platelets (Ara et al., 2001). Thus selectins $P$ and selectins $E$ which bind E-selectin ligand-1 (ESL-1) on leukocytes and stimulate rolling and adhesion leukocytes to the endothelium play an important role in the atherosclerotic process. Moreover, the activated endothelial cells can release von Willebrand factor (vWF) into the subendothelial matrix which, together with PAF promote secretion of ATP, ADP, and serotonin by platelets, their aggregation and adhesion to the subendothelium, playing important role in atherosclerosis (Zhang et al., 2010). Endothelin produced primarily in the endothelium and thromboxane produced by activated platelets that play a key role in vascular homeostasis and platelets aggregation are also crucial for atherosclerosis. The above-mentioned processes of endothelial dysfunction and activation together with lipids disturbances, manifested by an increase of modified, i.e. oxidized forms of low density of lipoproteins (ox-LDL cholesterol) are key for the atherosclerotic plaque formation. 


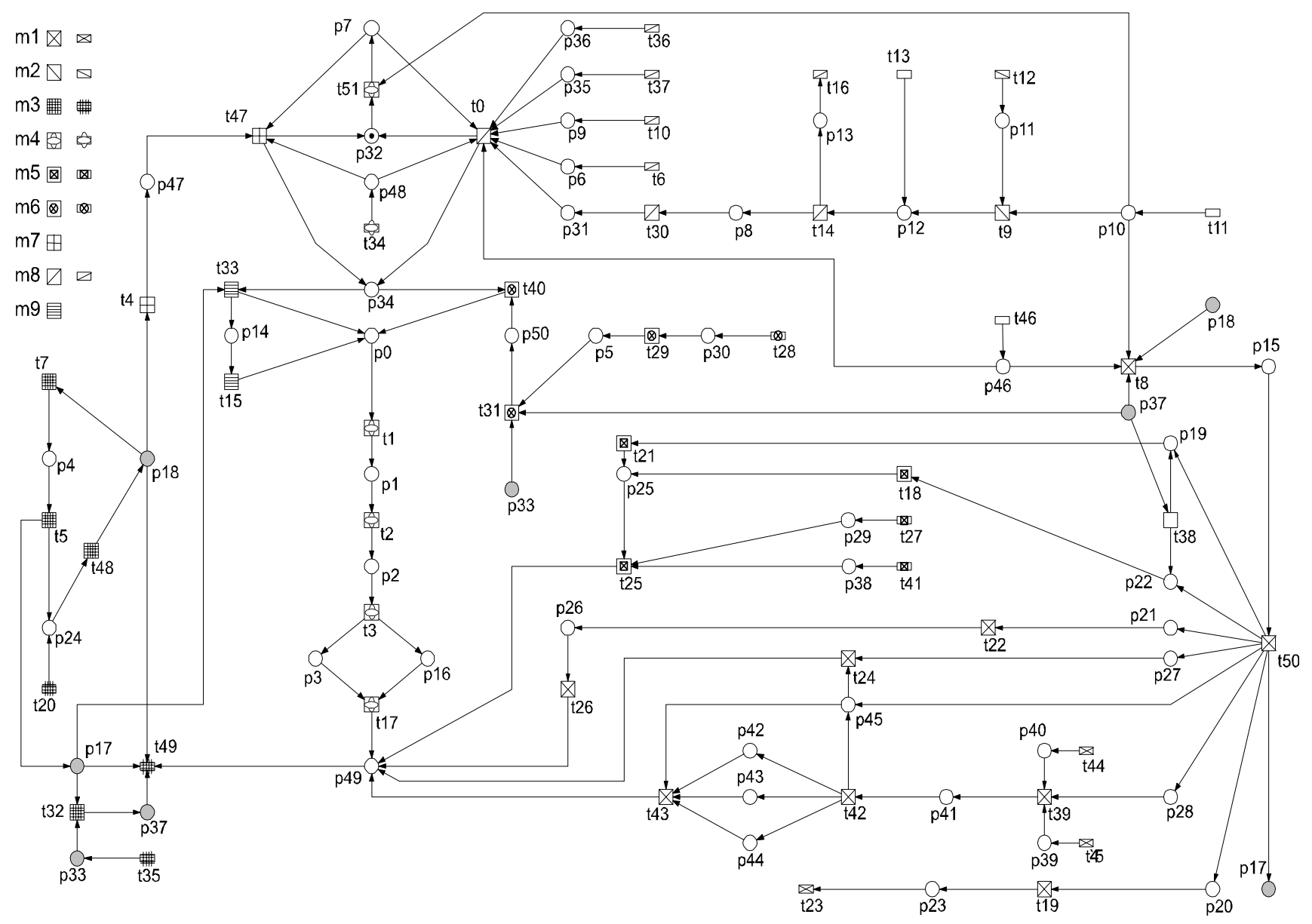

Fig. 1. Model with transitions marked by MCT-sets

\section{The Petri net based model and its analysis}

The biological process described in the previous subsection has been modeled as a Petri net. The net, presented in Figure 1, contains 51 places and 52 transitions. The biological meaning of the places is described in Table 1 while the meaning of the transitions is provided in Table 2 . The net contains 8 minimal t-invariants. Since there are no read arcs in the net, all these invariants are feasible. It is worth mentioning that it could be expected that the net of this size contains much more minimal t-invariants. Such a small number of minimal $\mathrm{t}$-invariants follows from the structure of the net, where, for example, there are two transitions ( $\mathrm{t} 0$ and $\mathrm{t} 50$ ) directly connected with quite a substantial number of places.

The net has 9 MCT-sets of size greater than 1 and 4 single transition MCT-sets. This means that t-invariants are constructed mainly of larger blocks of shared subprocesses. The MCT-sets are listed in Table 3 and are depicted in Figure 1. The list of minimal t-invariants is presented in Table 4.

It is easy to observe that MCT-set $\mathrm{m} 3$ is common for all t-invariants and it contains transitions responsible for the inflammatory process. It can also be observed that MCT-set $\mathrm{m}$ 4, which contains core transitions of NO regulation, is involved in almost all t-invariants (except i1 and i2). This group has two subgroups - one based on MCT-set $\mathrm{m} 6$ and one based on MCT-set $\mathrm{m} 9$. These two groups represent alternative paths of lowering $\mathrm{NO}$ - one dependent on reactive oxygen species (ROS) and the other dependent on the asymmetric dimethylarginine (ADMA).

T-invariants which are not based on NO regulation (MCT-set m4) rely on the immune system response for a higher level of oxidized LDL cholesterol (MCT-set m5). One of them (i2) relies on processes related to endothelium (MCT-set m1) and the other (i1) does not involve endothelium activation. 
Table 1. The IDs and names of the places of the model

\begin{tabular}{|c|c|c|c|}
\hline No. & Place name & No. & Place name \\
\hline $\mathrm{P} 0$ & low NO in endothelium & $\mathrm{p} 26$ & selectins P PSGL1 complex \\
\hline $\mathrm{P} 1$ & low cGMP increase & $\mathrm{p} 27$ & increased endothelin \\
\hline $\mathrm{P} 2$ & low activated $\mathrm{Ca}$ ATPase & $\mathrm{p} 28$ & PAF synthesis \\
\hline P3 & low vasodilatation & $\mathrm{p} 29$ & CD31 receptor \\
\hline $\mathrm{P} 4$ & activated macrophages & p30 & PRMT \\
\hline P5 & free ADMA & $\mathrm{p} 31$ & eNOS \\
\hline $\mathrm{P} 6$ & $\mathrm{NADPH}$ & p32 & citrulline \\
\hline $\mathrm{P} 7$ & L-arginine & p33 & LDL cholesterol \\
\hline P8 & $\mathrm{CaM}$ & p34 & NO \\
\hline P9 & $\mathrm{BPH} 4$ & p35 & bradykinin \\
\hline $\mathrm{p} 10$ & increased shear stress & p36 & Ach \\
\hline $\mathrm{p} 11$ & vasodilators agonists & p37 & ox-LDL cholesterol \\
\hline $\mathrm{p} 12$ & CaM caveolin 1 complex & p38 & Intracellular proteins \\
\hline $\mathrm{p} 13$ & caveolin 1 & p39 & vWF exposed \\
\hline $\mathrm{p} 14$ & $\mathrm{OONO}^{-}$-anion peroxynitrite & $\mathrm{p} 40$ & collagen exposed \\
\hline $\mathrm{p} 15$ & endothelium activated & $\mathrm{p} 41$ & platelets adhesion to endothelium \\
\hline $\mathrm{p} 16$ & high vasoconstriction & $\mathrm{p} 42$ & $\mathrm{ADP}$ \\
\hline $\mathrm{p} 17$ & ROS & $\mathrm{p} 43$ & serotonin \\
\hline $\mathrm{p} 18$ & cytokines & $\mathrm{p} 44$ & ATP \\
\hline $\mathrm{p} 19$ & ICAM 1 & $\mathrm{p} 45$ & increased tromboxane \\
\hline p20 & selectins $\mathrm{E}$ & $\mathrm{p} 46$ & endothelium \\
\hline $\mathrm{p} 21$ & selectins $\mathrm{P}$ & $\mathrm{p} 47$ & iNOS \\
\hline $\mathrm{p} 22$ & VCAM 1 & $\mathrm{p} 48$ & hydrogen peroxide \\
\hline $\mathrm{p} 23$ & selectins E-ESL1 complex & $\mathrm{p} 49$ & atherosclerosis \\
\hline $\mathrm{p} 24$ & inflammation & $\mathrm{p} 50$ & increased ADMA \\
\hline $\mathrm{p} 25$ & leukocytes adhesion & & \\
\hline
\end{tabular}

The minimal t-invariants are grouped into t-clusters using UPGMA algorithm. The resulting dendrogram presented in Figure 2 describes the dependencies among the subprocesses corresponding to MCT-sets.

The analysis of the net verified the accuracy of the model. The net is a basis for future research where the model will be extended and complemented by available qualitative information. After that it will be used to answer some open questions concerning the modeled biological process. The model will be extended by adding atherosclerotic predisposing risk factors such as smoking, wrong diet, diabetes mellitus, metabolic syndrome and checking their influence on the model behavior and selected subprocesses. This will also help in studying the direct influence of these factors on the discussed processes.

\section{Conclusions}

In this paper, a Petri net based model of some parts of the process of endothelial dysfunction has been presented. In particular, the model contains information about the relations between this process and atherosclerosis. The presented model is quantitative, which means that it describes the topology of the network of interrelations among the basic components of the analyzed process. In many cases, in the area of biological systems, models of this type are very important since the structure of such a system is crucial for its behavior. 
Table 2. The IDs and names of transitions of the model

\begin{tabular}{|c|c|c|c|}
\hline No. & Transition name & No. & Transition name \\
\hline to & NO synthesis by eNOS & $\mathrm{t} 26$ & leukocytes uptake \\
\hline $\mathrm{t} 1$ & $\begin{array}{l}\text { low NO diffuses from endothelium } \\
\text { to vascular smooth muscles }\end{array}$ & $\mathrm{t} 27$ & CD31 synthesis \\
\hline $\mathrm{t} 2$ & low protein kinase $\mathrm{G}$ activation & $\mathrm{t} 28$ & protein synthesis \\
\hline $\mathrm{t} 3$ & low calcium active uptake & $\mathrm{t} 29$ & Proteolysis \\
\hline $\mathrm{t} 4$ & iNOS synthesis activation & $\mathrm{t} 30$ & eNOS synthesis \\
\hline t5 & processes leading to ROS increase & $\mathrm{t} 31$ & processes increasing ADMA synthesis \\
\hline t6 & NADPH synthesis & $\mathrm{t} 32$ & LDL modification \\
\hline $\mathrm{t} 7$ & macrophages activation & $\mathrm{t} 33$ & processes with ROS lowering NO concentration \\
\hline t8 & endothelium activation & $\mathrm{t} 34$ & oxygen synthesis \\
\hline $\mathrm{t} 9$ & intracellular calcium increase & $\mathrm{t} 35$ & LDL cholesterol synthesis \\
\hline $\mathrm{t} 10$ & BPH4 synthesis from GTP & $\mathrm{t} 36$ & Ach synthesis \\
\hline $\mathrm{t} 11$ & shear stress processes & $\mathrm{t} 37$ & bradykinin synthesis \\
\hline $\mathrm{t} 12$ & vasodilators agonists synthesis induction & $\mathrm{t} 38$ & stimulation \\
\hline $\mathrm{t} 13$ & CaM caveolin-1 binding & $\mathrm{t} 39$ & platelets endothelial adhesion processes \\
\hline $\mathrm{t} 14$ & Cleavage & $\mathrm{t} 40$ & processes with ADMA lowering NO concentration \\
\hline $\mathrm{t} 15$ & nitrosylation & $\mathrm{t} 41$ & intracellular proteins synthesis \\
\hline $\mathrm{t} 16$ & caveolin usage & $\mathrm{t} 42$ & factor secretion by platelets \\
\hline $\mathrm{t} 17$ & vasoconstriction process caused by low NO & $\mathrm{t} 43$ & further platelets activation \\
\hline $\mathrm{t} 18$ & adhesion VCAM1 & $\mathrm{t} 44$ & collagen exposition \\
\hline $\mathrm{t} 19$ & binding with ESL1 ligands on leukocytes & $\mathrm{t} 45$ & vWF exposition \\
\hline $\mathrm{t} 20$ & inflammatory process & $\mathrm{t} 46$ & endothelial synthesis \\
\hline $\mathrm{t} 21$ & ICAM1adhesion & $\mathrm{t} 47$ & NO synthesis by iNOS \\
\hline $\mathrm{t} 22$ & binding with PSGL 1 ligands on leukocytes & $\mathrm{t} 48$ & cytokines synthesis \\
\hline $\mathrm{t} 23$ & leukocytes rolling and adhesion to endothelium & $\mathrm{t} 49$ & increase in atheroslerosis processes \\
\hline $\mathrm{t} 24$ & $\begin{array}{l}\text { vasoconstriction process induced by endothelin } \\
\text { and tromboxane }\end{array}$ & $\mathrm{t} 50$ & substances synthesized by activated endothelium \\
\hline $\mathrm{t} 25$ & leukocytes adhesion and transmigration & $\mathrm{t} 51$ & reaction catalyzed by DDAH \\
\hline
\end{tabular}

Table 3. The MCT-sets of the model

\begin{tabular}{l|l|l}
\hline $\begin{array}{c}\text { MCT } \\
\text { No. }\end{array}$ & \multicolumn{1}{|c}{ Transition list } & \multicolumn{1}{c}{ Biological meaning } \\
\hline $\mathrm{m} 1$ & $\mathrm{t} 8, \mathrm{t} 19, \mathrm{t} 22, \mathrm{t} 23, \mathrm{t} 24, \mathrm{t} 26, \mathrm{t} 39, \mathrm{t} 42, \mathrm{t} 43, \mathrm{t} 44, \mathrm{t} 45, \mathrm{t} 50$ & processes associated with endothelial activation \\
\hline $\mathrm{m} 2$ & $\mathrm{t} 0, \mathrm{t} 6, \mathrm{t} 10, \mathrm{t} 14, \mathrm{t} 16, \mathrm{t} 30, \mathrm{t} 36, \mathrm{t} 37$ & processes involved in NO synthesis \\
\hline $\mathrm{m} 3$ & $\mathrm{t} 5, \mathrm{t} 7, \mathrm{t} 20, \mathrm{t} 32, \mathrm{t} 35, \mathrm{t} 48, \mathrm{t} 49$ & $\begin{array}{l}\text { processes enhancing atherosclerotic plaque formation (inflam- } \\
\text { mation and oxidative stress) }\end{array}$ \\
\hline $\mathrm{m} 4$ & $\mathrm{t} 1, \mathrm{t} 2, \mathrm{t} 3, \mathrm{t} 17, \mathrm{t} 34, \mathrm{t} 51$ & processes leading to vasoconstriction of the vessels \\
\hline $\mathrm{m} 5$ & $\mathrm{t} 18, \mathrm{t} 21, \mathrm{t} 25, \mathrm{t} 27, \mathrm{t} 41$ & $\begin{array}{l}\text { processes influencing leukocytes behavior in atherosclerotic } \\
\text { process }\end{array}$ \\
\hline $\mathrm{m} 6$ & $\mathrm{t} 28, \mathrm{t} 29, \mathrm{t} 31, \mathrm{t} 40$ & ADMA influence on NO synthesis \\
\hline $\mathrm{m} 7$ & $\mathrm{t} 4, \mathrm{t} 47$ & processes influencing iNOS \\
\hline $\mathrm{m} 8$ & $\mathrm{t} 9, \mathrm{t} 12$ & processes influencing intracellular calcium level \\
\hline $\mathrm{m} 9$ & $\mathrm{t} 15, \mathrm{t} 33$ & nitrosylation \\
\hline
\end{tabular}


Table 4. t-invariants of the model as a sum of MCT-sets

\begin{tabular}{c|l|l}
\hline t-invariant No. & \multicolumn{1}{|c|}{ MCT sets } & \multicolumn{1}{|c}{ Single transitions } \\
\hline i1 & $\mathrm{m} 3, \mathrm{~m} 5$ & $\mathrm{t} 38$ \\
\hline $\mathrm{i} 2$ & $\mathrm{~m} 1, \mathrm{~m} 3, \mathrm{~m} 5$ & $\mathrm{t} 11, \mathrm{t} 46$ \\
\hline $\mathrm{i} 3$ & $\mathrm{~m} 2, \mathrm{~m} 3, \mathrm{~m} 4, \mathrm{~m} 9$ & $\mathrm{t} 11, \mathrm{t} 13, \mathrm{t} 46$ \\
\hline $\mathrm{i} 4$ & $\mathrm{~m} 2, \mathrm{~m} 3, \mathrm{~m} 4, \mathrm{~m} 6$ & $\mathrm{t} 11, \mathrm{t} 13, \mathrm{t} 46$ \\
\hline i5 & $\mathrm{m} 2, \mathrm{~m} 3, \mathrm{~m} 4, \mathrm{~m} 8, \mathrm{~m} 9$ & $\mathrm{t} 11, \mathrm{t} 46$ \\
\hline $\mathrm{i} 6$ & $\mathrm{~m} 2, \mathrm{~m} 3, \mathrm{~m} 4, \mathrm{~m} 6, \mathrm{~m} 8$ & $\mathrm{t} 11, \mathrm{t} 46$ \\
\hline i7 & $\mathrm{m} 3, \mathrm{~m} 4, \mathrm{~m} 7, \mathrm{~m} 9$ & $\mathrm{t} 11$ \\
\hline i8 & $\mathrm{m} 3, \mathrm{~m} 4, \mathrm{~m} 6, \mathrm{~m} 7$ & $\mathrm{t} 11$ \\
\hline
\end{tabular}

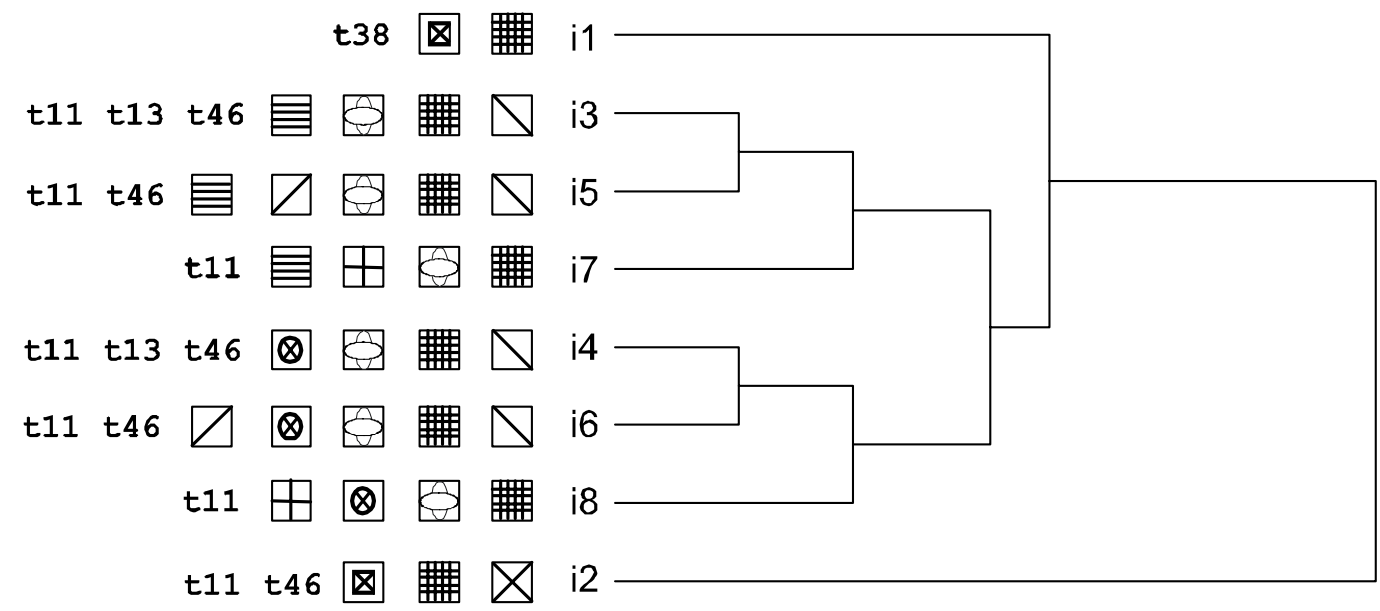

Fig. 2. t-invariant clusters illustrated as a sum of MCT-sets. Clustering method is UPGMA with Euclidean distances metric. t-invariants are represented as 52-dimensional vectors (due to total 52 transitions)

Moreover, construction of an exact quantitative model, e.g. one based on differential equations, of biological system is usually impossible since not all parameters necessary in such a model are known (i.e. the knowledge about quantitative aspects of a biological system is usually very limited). When the model is expressed in the language of Petri net theory, their qualitative version can be relatively easily complemented by quantitative data when they become available. In order to do this, some Petri net extensions can be used.

The model presented in this paper has been analyzed using the methods based on invariant analysis. The p- and t-invariants have been calculated and MCT-sets and t-clusters have been determined.

This model is a starting point for future research. Among others, the work will be extended by taking into account the role of the typical cardiovascular risk factors in the process of atherosclerosis development. It would be also very interesting to collect and include some quantitative data in the model, especially information on the quantities of the substances involved in the reactions modeled by transitions and the time dependencies. The extended model will be used to test some hypotheses concerning the modeled biological system.

\section{References}

Ara J., Mirapeix E., Arrizabalaga P., Rodriguez R., Ascaso C., Abellana R., Font J., Darnell A. (2001) Circulating soluble adhesion molecules in ANCA-associated vasculitis. Nephrol. Dial. Transplant. 16: 276-285.

Cheng P.F., Tsai A., Berka V., Wu K. (1997) Mutation of Glu361 in human endothelial nitric-oxide synthase selectively abolishes L-arginine binding without perturbing the behaviour of heme and other redox centers. J. Biol. Chem. 272: 6114-6118. 
Cooke J. (2000) Does ADMA cause endothelial dysfunction? Arterioscler. Thromb. Vasc. Biol. 20: 2032-2037.

Davignon J., Ganz P. (2004) Role of endothelial dysfunction in atherosclerosis. Circulation 109: 27-32.

de Haro Miralles J., Martinez-Aguilar E., Florez A., Varela C., Bleda S., Acin F. (2009) Nitric oxide: link between endothelial dysfunction and inflammation in patients with peripheral arterial disease of the lower limbs. Interact. CardioVasc. Thorac. Surg. 9: 107-112.

Endemann D., Schiffrin E. (2004) Endothelial dysfunction. J. Am. Soc. Nephrol. 15: 1983-1992.

Formanowicz D., Sackmann A., Kozak A., Blazewicz J., Formanowicz P. (2011) Some aspects of the anemia of chronic disease modeled and analyzed by Petri net based approach. Bioproc. Biosys. Eng. 34: 581-595.

Grafahren-Belau E., Schreiber F., Heiner M., Sackmann A., Bjorn H.J., Grunwald S., Speer A., Windre K., Koch I. (2008) Modularization of biochemiical networks based on classification of Petri net t-invariants. BMC Bioinformatics 9: 90 .

Koch I., Reisig W., Schreiber F. (2011) Modeling in Systems Biology. The Petri Net Approach. Springer, London.

Murata T. (1989) Petri nets: Properties, analysis and applications. Proc. IEEE 77: 541-580.

Petri C.A. (1962) Communication with automata. Insitut fur Automata Schriften des IIM .
Reisig W. (1985) Petri nets: an introduction. Springer-Verlag, Berlin.

Reisig W. (2011) Petri nets. In: Modeling in Systems Biology. The Petri Net Approach, ed. I. Koch, W. Reisig, F. Schreiber, Springer, London, pp. 37-56.

Sackmann A. (2011) Discrete modeling. In: Modeling in Systems Biology. The Petri Net Approach, ed. I. Koch, W. Reisig, F. Schreiber, Springer, London, pp. 59-72.

Sackmann A., Formanowicz D., Formanowicz P., Koch I., Blazewicz J. (2007) An analysis of the Petri net based model of the human body homeostasis process. Comput. Biol. Chem. 31: 1-10.

Sackmann A., Heiner M., Koch I. (2006) Application of Petri net based analysis techniques to signal transduction pathway. BMC Bioinformatics 7: 482.

Sessa W., Harrison J., Barber C., Zeng D., Durieux M., DAngelo D., Lynch K., Peach M. (1992) Molecular cloning and expression of a cDNA encoding endothelial cell nitric oxide synthase. J. Biol. Chem. 267: 5274-5276.

Vallance P., Leone A., Calver A., Collier J., Moncada S. (1992) Accumulation of an endogenous inhibitor of NO synthesis in renal failure. Lancet 339: 572-575.

Zhang J., DeFelice A., Hanig J., Colatsky T. (2010) Biomarkers of endothelial cell activation serve as potential surrogate markers for drug-induced vascular injury. Toxicol. Pathol. 38: 856-871. 